\title{
Predictors of Obesity among Adults Who Have Diabetes Mellitus Type Two
}

\author{
Reem Ahmad Jarrad ${ }^{*}$, Zyad Saleh1, Naser Mahmoud ${ }^{2}$ \\ ${ }^{1}$ Clinical Nursing Department, School of Nursing, The University of Jordan, Amman, Jordan \\ ${ }^{2}$ Jordan Ministry of Health, Amman, Jordan \\ Email:*r.jarrad@ju.edu.jo, zyad.saleh2@ju.edu.jo,n.mahmoud1989@yahoo.com
}

How to cite this paper: Jarrad, R.A., Saleh, Z. and Mahmoud, N. (2018) Predictors of Obesity among Adults Who Have Diabetes Mellitus Type Two. Open Journal of Nursing, 8, 580-589.

https://doi.org/10.4236/ojn.2018.88043

Received: July 26, 2018

Accepted: August 25, 2018

Published: August 28, 2018

Copyright (๑) 2018 by authors and Scientific Research Publishing Inc. This work is licensed under the Creative Commons Attribution International License (CC BY 4.0).

http://creativecommons.org/licenses/by/4.0/ (c) (i) Open Access

\begin{abstract}
Background and Purpose: Obesity and Diabetes Mellitus type two (DMII) have a known association. Yet, the socio-demographic predictors of obesity in special populations such as people who have DMII remain unclear. The purpose of this study was to determine the socio-demographic predictors of obesity among adults who have DMII. Materials and Methods: This was a descriptive cross-sectional study targeting 488 adult clients who had the diagnosis of DMII. The participants were asked to complete a survey covering demographic and clinical variables of age, gender, employment, income, education, weight, height, medical insurance, duration of diabetes and type of treatment taken to control diabetes. Besides, Body Mass Index (BMI), the dependent variable, was calculated. Descriptive statistics were used to present clients' socio-demographic and clinical characteristics. Univariate Binary logistic regression was used to determine the socio-demographic predictors of obesity. Results: Results showed that age, household income and employment were independent predictors of BMI in adults who have DMII. Gender and level of education were not significant predictors of higher BMI. Conclusion: Those results suggest that understanding of the contributing variables of obesity in adults who have DMII can help identify the at-risk groups allowing for early diagnosis and establishment of effective prevention and management plans.
\end{abstract}

\section{Keywords}

Obesity, Diabetes, Predictors

\section{Introduction}

Diabetes global prevalence among adults over 18 years has risen from $4.7 \%$ in 1980 to $8.5 \%$ in 2014 . The number of people who have diabetes was 422 million 
in 2014, $90 \%$ of them have DMII [1]. In middle and low income countries, the number of people who have DMII is increasing exponentially and is considered an eminent threat on healthcare systems and resources [2]. Alongside, Diabetes has many serious long term health consequences such as retinopathy with potential loss of vision; nephropathy, peripheral and central neuropathy, acute coronary syndrome, hypertension and cerebrovascular diseases [3].

Major risk factors of DMII include positive family history, race, older age, high blood pressure, dyslipidemia, inactivity and sedentary life style [4]. Many of these factors are connected to obesity which can explain, in part, the association between obesity and DMII [5]). It is estimated that about $90 \%$ of people who have DMII aged (16 - 54) years have overweight or obesity which is seriously indicative of a problem in that area [6]. In adults who have overweight or obesity and are at risk for DMII, lifestyle modifications can reduce 2.5 to $5.5 \mathrm{~kg}$ of body weight in two or more years, cutting the incidence of DMII by $30 \%$ to $60 \%$ [7] [8]. Those findings suggest that weight reduction measures are a necessity not only for people who have DMII but also for those at risk [9].

This research examined the socio-demographic predictors of obesity in adults who have DMII because of the apparent swinging when looking into the available evidence. Correlations between weight and studied socio-demographic variables like age, gender, education, income and employment were not, most of the time, a clear cut positive or negative. On the contrary, correlations were redundant when different populations/population slices were studied or when different population characteristics were surveyed and their moderating effects were entered into the correlation equations [10] [11]. Worth telling, that this is a precedent study in terms of focusing attention on obesity predictors in adults with DMII. In fact, identifying predictors of obesity in special populations can guide policy makers to lead timely and wise health resource utilization and disease prevention efforts.

The study purpose was to determine whether socio-demographic characteristics including age, gender, education, income and current employment status can predict obesity in adults who have DMII after controlling for having medical insurance, duration of diabetes mellitus and type of prescribed medical treatment. No hypotheses were set due to lack of supportive consistent literature in that research area.

\section{Materials and Methods}

\subsection{Subjects and Study Design}

This was a descriptive cross-sectional study targeting adult clients who have DMII. It was conveniently sampled and included 488 Jordanian participants who were recruited from diabetes outpatient clinics in four major cities which are: Amman, Zarqa, Russeifah and Mafraq between June 2015 and November 2016. Sample size was calculated in accordance with univariate binary logistic regression tables. The participants were twenty years or above, Arabic literate and had a confirmed diagnosis of DMII. There was an eligibility check carried out by re- 
ferring to the medical file to make sure that the participants had the diagnosis of DMII. The eligible subjects were asked to sign a consent form after having a detailed explanation about the purpose of the study.

\subsection{Measures}

The participants were asked to complete a demographic/clinical data sheet. The sheet is single page, phrased in Arabic, reviewed by clinical and language specialists, checked for face validity by piloting, anonymous, and only numbered for the documentation purpose. It included variables of age, gender, employment, income, education, weight, height, medical insurance, duration of diabetes and the type of treatment taken to control diabetes. The participants were recruited from the internal medicine, endocrinology and diabetes clinics during the waiting time. The eligible approving participants were accompanied to a calm spot near the waiting room and filled the demographic/clinical data sheet. The process took five minutes. The confidentiality, right to withdraw and no harm principles were assured throughout the process.

After data sheets were filled, the participants weight was measured using a properly functioning weighing machine and length was taken by a standard measuring tape in order to calculate and document their Body mass index (BMI). BMI can be used for population assessment of overweight and obesity in variable medical contexts. This is because BMI calculation requires only height and weight; it is inexpensive and easy to use for clinicians and for the general public [12]. BMI is calculated by taking a person's weight in kilograms and dividing it by the person's squared height in meters. Obesity is defined as BMI greater than or equal to $30 \%$ [13].

\subsection{Data Analysis}

SPSS software for Windows version 17.0 was used for data analysis (SPSS Inc., Chicago, Illinois). Descriptive statistics were used to present clients' socio-demographic and clinical characteristics. Data were summarized using frequencies and percentages for categorical variables while means and standard deviations were used to describe continuous variables.

Binary logistic regression was used to determine the socio-demographic predictors (age, gender, level of education income and employment status) of obesity after controlling for having medical insurance, duration of diabetes mellitus and type of prescribed medical treatment. All socio-demographic and clinical variables were entered together into the binary logistic regression analysis in one block. A p-value of $\leq 0.05$ was used to determine statistical significance.

\section{Results}

\subsection{Sample Characteristics}

Socio-demographic and clinical characteristics of clients are presented in Table 1. Their average age was $60 \pm 10.2$ years (range 23 - 89) and around half of them 
Table 1. Patients' Characteristics $(n=488)$.

\begin{tabular}{lc}
\hline Age (years) & $60.0 \pm 10.2$ \\
Male & $261(53.5)$ \\
Houshold income* & \\
Low income & $390(79.9)$ \\
Average income or higher & $98(21.1)$ \\
Level of education & \\
Less than high school & $243(49.8)$ \\
High school & $167(34.2)$ \\
Associate degree and higher & $78(16)$ \\
Employed & $115(23.6)$ \\
Having medical insurance & $382(78.3)$ \\
Weight (kilogram) & $82.1 \pm 13.5$ \\
Body mass index $\geq 30$ kg/m ${ }^{2}$ & $210(43.0)$ \\
Medical treatment of diabetes mellitus & \\
Oral hypoglycemic & $318(65.2)$ \\
Insullin injection & $94(19.3)$ \\
Oral hypoglycemic and insullin injection & $76(15.6)$ \\
Duration of diabetes mellitus & $10.4 \pm 7.1$ \\
\hline
\end{tabular}

Data are presented as mean $\pm \mathrm{SD}$ or $\mathrm{n}(\%) .{ }^{*}$ Income level: low income is less than 10 dollars/day and average income or higher is more than 11 dollars/day.

were males. Most of clients had medical insurance, were unemployed and fell within the low household income category. Majority of clients had education less than high school, around one third had high school education and only $16 \%$ had associate degree or higher education. Slightly less than half of clients had obesity. The average duration of diabetes mellitus was $10.4 \pm 7.1$ years. Majority of clients were prescribed oral hypoglycemic agents; while nearly one third were prescribed only insulin injection or combined treatment of oral hypoglycemic agents and insulin.

\subsection{Prediction of Obesity}

Socio-demographic predictors of higher BMI (after controlling for having medical insurance, duration of diabetes mellitus and medical treatment of diabetes mellitus) are presented in Table 2. Age, household income and employment status were independent predictors of BMI. For every 1-year increase in age, clients were $3 \%$ less likely to have obesity. Clients with low household income were 2 times more likely to have obesity compared to clients with an average or higher household income. Unemployed clients had 2.3 times higher chances to have obesity compared to employed ones. Gender and level of education were not significant predictors of higher BMI. 
Table 2. Socio-demographic predictors of obesity $(n=488)$.

\begin{tabular}{lcccc}
\hline Predictors of obesity & Odd ratio & \multicolumn{2}{c}{$95 \%$ CI } & P value \\
\hline Duration of diabetes mellitus & 0.96 & 0.92 & 0.99 & 0.008 \\
Medical treatment of diabetes mellitus ${ }^{1}$ & & & & \\
Oral hypoglycemic & 2.7 & 1.55 & 4.8 & 0.001 \\
Insulin injection & 0.44 & 0.22 & 0.86 & 0.02 \\
Having insurance & 1.15 & 0.70 & 1.9 & 0.58 \\
Age & 0.97 & 0.95 & 0.99 & 0.004 \\
Gender (female) & 0.71 & 0.46 & 1.09 & 0.12 \\
Level of education & 0.87 & 0.65 & 1.17 & 0.36 \\
$\begin{array}{l}\text { Household income } \\
\text { (Average household income or higher) }\end{array}$ & 0.51 & 0.33 & 0.80 & 0.004 \\
Employment status (unemployed) & 2.3 & 1.3 & 4.0 & 0.003 \\
\hline
\end{tabular}

CI: confidence interval. ${ }^{1}$ Reference group: all other types of diabetes mellitus treatment; Dummy coded variable; medical treatment with oral hypoglycemic and insulin injection.

\section{Discussion}

\subsection{Gender and Obesity}

Gender was not a BMI predictor in adults who had DMII. This finding was relatively inconsistent with previous general population studies which linked BMI positively with female gender in certain socioeconomic and/or some age groups [14]. It is apparent that some factors interfere with the effect of gender on weight such as age, socially patterned exposures occurring in childhood and adolescence, marital status, level of education, household income and time spent in physical activity [15] [16]. It could be concluded that socioeconomic, lifestyle and cultural factors may be of modifying effect ameliorating the obesity predictive effect of gender.

\subsection{Education and Obesity}

Education was not a significant predictor of BMI among adults who had DMII. This finding was not quite consistent with the general population studies where the association between BMI and education was significant but redundant between positive and negative per surveyed socioeconomic stratum of developed versus developing countries [17] [18] [19]. Those discrepancies may be attributed, at least partially, to socio-economic status (SES) variability of different societies, different samples and even different studied slices taken from same society. Such indefinite number of SES factors that couldn't be always surveyed, possibly have dragging effect towards positive trends or negative trends or even towards a cancelling effect that could explain the non-predictive power of education for BMI in adults who had DMII.

\subsection{Age and Obesity}

Age was a remarkable demographic variable as for every 1-year increase in age; 
clients were 3\% less likely to have obesity. This finding was contradictory when compared to the general, possibly not having diabetes, populations whose trends were positive [17] [20]. Our primary result could be attributed, at least in part; to "Diabetes life style self management" measures that those clients are probably educated about and adhered to, especially when they get older and more committed with their life and health choices [21] [22]. Besides, the use of oral hypoglycemic agents especially metformin (glucophage) for longer duration of time could have interfered in weight lowering effect [23]. This explanation doesn't cancel the need to conduct a longitudinal repeated measures design to track the stability of the correlation between age and BMI over time in Adults who have DMII.

\subsection{Income and Obesity}

Clients with low household income were 2 times more likely to have obesity compared to those with an average or higher household income. Relatively inconsistant, BMI was positively associated with socioeconomic status, in a slice of low-income general population of adults [18]. This evidence was found in a selectively poor community, so the higher socioeconomic status stratae can be still considered poor in comparison to other population slices, hence rejecting the apparent inconsistancy. Supposedly, the view that people in poverty are less healthy in terms of excess accumulation of fat persists as a coventional wisdom. The previous assumption reflects important differences in the relationship between income and BMI. While people living in poverty are expected to have higher BMIs, for those at the tails of the BMI distribution, increases in income are correlated with healthier BMI values [24]. The direct simple conclusion of our study suggests that the conventional wisdom still applies to the Jordanian people who have DMII in a comparative way to the general population who have no diabetes. It is recommended to activate targeted community based or clinic based health programs aimed at raising awareness and encouraging life style, weight, physical activity and diet changes among the lower income risky groups who have DMII.

\subsection{Employment and Obesity}

Unemployed clients were 2.3 times more likely to have obesity when contrasted to employed ones. In comparison, a large scale general population evidence reported that employment status showed varying impacts on obesity by age and gender. Both unemployment at or after 60 , as well as unemployment among women, were associated with increased BMI compared with unemployment among younger individuals or men, respectively [25]. Those results are aligned in general with our finding. It could be added that those who don't work may tend to be less physically active, eat more carbohydrates, watch more televesion among other sedentary life practices, the thing that makes them more vulnerable to obesity than the employed ones [26]. It is recommended for the health au- 
thority to target the off employment populations who have DMII with purposeful physical activity "out door programs" to diminish the "stay at home" negative effect on weight.

\section{Recommendations}

The present study is particularly valuable in that it provides evidence for the need of clinic-centered and community-centred culturally sensitive healthcare provisions, which maximizes the role of the health care authorities in primary prevention and detection, the thing that is critical in reducing obesity and obesity disparities in adults who have DMII. Thus, it leads to significant reductions in health care costs and related morbidities, complications, burdens, disabilities and mortalities.

\section{Conclusion}

It is clinically significant to survey the socio-demographic variables of adult clients who have DMII. Such data can enlighten the health authority on when and for whom to focus attention when managing parallel obesity and Diabetes issues.

\section{Limitations}

Convenient sampling had let certain sample characteristics to be prevailing such as unemployment, lower socioeconomic stratae, poorer incomes, less higher education, higher BMIs, etc. Besides, military and private hospital clients were not included nor adults who have DMII and are treated with diatary regimen only. Hence, future replication studies are advised to adopt randomization in order to enhance the generalizability of the findings.

\section{Declaration of Conflict of Interest}

The authors declare no conflict of interest of any kind.

\section{Funding}

This work is funded by the Deanship of Academic Research in The University of Jordan.

\section{Authorship Consent}

The authors allow the Journal to fulfill its publishing policy upon acceptance. Hereby, we confirm that this research work is original and has never been published elsewhere by any mean. All authors agreed on the current paper publishable format.

\section{Authors' Contributions}

The principal author RA contributed to the major concept and design building, 
preparation of the manuscript and coordinating research process. ZS contributed to the statistical analysis and results section. While NM contributed to data acquisition and validation processes.

\section{Acknowledgements}

We thank the generous contributions and support of the following parties: The University of Jordan, The University of Jordan Hospital, The Ministry of Health in Jordan and Dr. Khalil Yousif from the Clinical Nursing Department in The University of Jordan.

\section{References}

[1] Wexler, D.J. (2017) Patient Education: Diabetes Mellitus Type 2: Overview (Beyond the Basics).

https://www.uptodate.com/contents/type-2-diabetes-overview-beyond-the-basics

[2] World Health Organization (WHO) (2016) Global Report on Diabetes. http://apps.who.int/h/iris/bitstream/10665/204871/1/9789241565257_eng.pdf

[3] American Diabetes Association (2010) Diagnosis and Classification of Diabetes Mellitus. Diabetes Care, 33, S62-S69.

[4] Mayo Clinic (2017) Diseases and Conditions: Diabetes Risk Factors. http://www.myoclinic.org/diseases-conditions/diabetes/basics/risk-factors/con-2003 3091

[5] Pi-Sunyer, X. (2009) The Medical Risks of Obesity. Postgraduate Medicine, 121, 21-33. https://doi.org/10.3810/pgm.2009.11.2074

[6] Gatineau, M., Hancock, C., Holman, N., Outhwaite, H., Oldridge, L., Christie, A., et al. (2014) Public Health England: Adult Obesity and Type 2 Diabetes. https://www.gov.uk/goverment/uploads/system/uploads/attachment_data/file/3389 34/Adult_obesity_and_type_2_diabetes_.pdf

[7] Center for Disease Control and Prevention (CDC) (2015) Living with Diabetes. http://www.cdc.gov/diabetes/living/index.html

[8] Wadden, T., Webb, V., Moran, C. and Bailer, B. (2012) Lifestyle Modification for Obesity: New Developments in Diet, Physical Activity, and Behavior Therapy. Circulation, 125, 1157-1170. https://www.ncbi.nlm.nih.gov/pmc/articles/PMC3313649/

[9] American College of Cardiology/American Heart Association Task Force on Practice Guidelines, Obesity Expert Panel (2014) Expert Panel Report: Guidelines (2013) for the Management of Overweight and Obesity in Adults. Obesity, 22, S41-S410. http://011055vdm.y.http.onlinelibrary.wiley.comju.proxy.coe-elibrary.com/doi/10.1 002/oby.20660/full

[10] Thomas, D., Weedermann, M., Fuemmeler, B., Martin, C., Dhurandhar, N., Bredlau, C., et al. (2014) Dynamic Model Predicting Overweight, Obesity, and Extreme Obesity Prevalence Trends. Obesity, 22, 590-597.

http://0l11h0d2j.y.https.onlinelibrary.wiley.com.ju.proxy.coe-elibrary.com/action/s howCitFormats?doi=10.1002\%2Foby.20520 https://doi.org/10.1002/oby.20520

[11] Smith, T., Marriott, B., Dotson, L., Bathalon, G., Funderburk, L., White, A., et al. (2012) Overweight and Obesity in Military Personnel: Sociodemographic Predictors. Obesity, 20, 1534-1538. 
http://0111h0d2t.y.https.onlinelibrary.wiley.com.ju.proxy.coe-elibrary.com/action/s howCitFormats?doi=10.1038\%2Foby.2012.25 https://doi.org/10.1038/oby.2012.25

[12] Lin, S., Naseri, T., Linhart, C., Morrell, S., Taylor, R., McGarvey, S.T., et al. (2017) Trends in Diabetes and Obesity in Samoa over 35 Years, 1978-2013. Diabetic Medicine, 34, 654-661. https://doi.org/10.1111/dme.13197

[13] Centres for Disease Control and Prevention (CDC) (2015) About Adult BMI. https://www.cdc.gov/healthyweight/assessing/bmi/adult_bmi/index.html

[14] Mendez, M., Monteiro, C. and Popkin, B. (2005) Overweight Exceeds Underweight among Women in Most Developing Countries. The American Journal of Clinical Nutrition, 81, 714-721. http://wwww.ncbi.nlm.nih.gov/pubmed/15755843

[15] Mujahid, M., Arbor, A., Roux, D., Borrell, L. and Nieto, F. (2005) Cross-Sectional and Longitudinal Associations of BMI with Socioeconomic Characteristics. Obesity Research, 13, 1412-1421. https://doi.org/10.1038/oby.2005.171

[16] MacFarlane, A., Abbott, G., Crawford, D. and Ball, K. (2009) Socio-Demographic and Behavioural Correlates of Weight among Women with Children Living in Socioeconomically Disadvantaged Neighbourhoods. International Journal of Obesity, 33, 1289-1298.

https://www.researchgate.net/publication/26781080_Sociodemographic_and_behav ioural_correlates_of_weight_among_women_with_children_living_in_socioecono mically_disadvantaged_neighborhoods https://doi.org/10.1038/ijo.2009.167

[17] Lenthe, V., Droomers, M., Schrijvers, C. and Mackenbach, J. (2000) Socio-Demographic Variables and 6 Year Change in Body Mass Index: Longitudinal Results from the GLOBE Study. International Journal of Obesity, 24, 1077-1084. https://doi.org/10.1038/sj.ijo.0801236

[18] Fernald, L. (2007) Socio-Economic Status and Body Mass Index in Low-Income Mexican Adults. Social Science \& Medicine, 64, 2030-2042. https://doi.org/10.1016/j.socscimed.2007.02.002

[19] Akinyemiju, T.F., Zhao, X., Sakhuja, S. and Jolly, P. (2016) Life-Course Socio-Economic Status and Adult BMI in Ghana; Analysis of the WHO Study on Global Ageing and Adult Health (SAGE). International Journal for Equity in Health, 15, 185. https://doi.org/10.1186/s12939-016-0474-x

[20] AlNsour, M., Al Kayyali, G. and Naffa, S. (2013) Overweight and Obesity among Jordanian Women and Their Social Determinants. Eastern Mediterranean Health Journal, 19, 1014-1019.

http://www.emro.who.int/emhj-vol-19-2013/12/overweight-and-obesity-among-jor danian-women-and-their-social-determinants.html

[21] Zoumenou, V., Himburg, S., Magnus, M., Johnson, P., Lobar, S. and Adoueni, V. (2009) Measure of Strength of Commitment to Successful Diabetes Self Management among Blacks: Reliability and Validity. European Journal of Scientific Research, 26, 176-188.

[22] Jarrad, R.A., Khalil, A. and Mahmoud, N. (2015) Psychometric Properties and Correlates of the Strength of Commitment to Life Style Self Management Instrument among Jordanians with Diabetes Mellitus Type II. The Journal of American Science, 11, 75-81. http://www.jofamericanscience.org/journals/am-sci/am111015/

[23] The Diabetes Prevention Program Research Group (2012) Long-Term Safety, Tolerability, and Weight Loss Associated With Metformin in the Diabetes Prevention Program Outcomes Study. Diabetes Care, 35, 731-737. 
https://doi.org/10.2337/dc11-1299

[24] Jolliffe, D. (2011) Overweight and Poor? On the Relationship between Income and the Body Mass Index. Economics \& Human Biology, 9, 342-355.

https://doi.org/10.1016/j.ehb.2011.07.004

[25] Noh, J., Kim, J., Park, J., Oh, I. and Kwon, Y. (2016) Age and Gender Differential Relationship between Employment Status and Body Mass Index among Middle-Aged and Elderly Adults: A Cross-Sectional Study. BMJ Open Journal, 6, e012117. http://bmjopen.bmj.com/content/bmjopen/6/11/e012117.full.pdf

[26] Crabtree, S. (2014) Obesity Linked to Long Term Unemployment in the US. http://www.gallup.com/poll/171683/obesity-linked-long-term-unemployment.aspx 